\title{
Editorial
}

\section{New TB Tools Need to be Affordable in the Private Sector: The Case Study of Xpert MTB/RIF}

Of the estimated 10.4 million people who fell ill with tuberculosis (TB) in 2016, only 6.3 million people were detected and officially notified to national TB programmes, leaving a gap of 4.1 million [1]. These 4.1 million people are considered 'missing.' Where are they and why are they missing?

A recent 13-country patient pathway analysis shows that about $60 \%$ of all TB patients seek care in the formal or informal private health sector [2]. Further, there is a striking correlation between the estimated number of missing patients in each country, and the proportion of care-seeking that is private.

In fact, the private health sector is a major source of health care in 12 countries with the highest TB burden, including India, Pakistan, the Philippines, Bangladesh, Afghanistan, Kenya, Uganda, Vietnam, Indonesia, Myanmar, Nigeria and Cambodia [3]. In these economies, even poor patients with TB seek care from private health care providers, and delayed diagnosis and misdiagnosis are widely recognized problems [4]. Further, patients as well as doctors do not respect public versus private boundaries and often switch between these sectors $[5,6]$.

So, if we want to diagnose TB early and accurately, we need to engage and work with the private health sector [3]. Regardless of where patients seek care, we need to ensure that they have access to high quality, patient-centric TB diagnosis and treatment [7]. This means that we need to make sure that new TB tools are not only scaled-up in the public sector, but are also affordable and accessible in the private health sector [8].

Xpert MTB/RIF (Cepheid Inc, Sunnyvale, USA), a WHO-endorsed rapid TB test, is the best front-line test we have today for TB detection and rapid drug-resistance screening [9]. This test is available at a concessional price of $\$ 9.98$ per cartridge. Data from Cepheid show that, as of 31 December 2017, a total of 9449 GeneXpert instruments and 34 million Xpert MTB/RIF cartridges had been procured in the public sector in 130 of the 145 countries eligible for concessional pricing.

Unfortunately, concessional pricing is restricted to the public sector in high burden countries. Private sector hospitals and laboratories are not eligible for concessional pricing, even if they are in highest TB burden countries. What is the impact of these restrictions on prices patients pay for Xpert MTB/RIF?

In 2015, we conducted the first study on pricing of Xpert MTB/RIF in the private sector in 12 highly privatized health markets [10].
As shown in the table below, in 6 of the 12 countries, there was no commercial availability of Xpert in the private sector [10]. In the remaining six countries, the average price charged by private laboratories or hospitals was US \$68.73 (range \$30.26-\$155.44), as compared to a fully loaded cost of $\$ 20-30$ per test paid by national TB programmes [10].

In 2017-18, we updated the survey to assess changes over time, using similar methods. Our new findings (Table 1) show that Xpert is now commercially available in the private sector in 7 of 12 countries. In comparison to the average price to the patient of US $\$ 68.73$ in 2015 , patients now pay US $\$ 84.53$ (range $\$ 46.70$ $\$ 175.00$ ), which translates to a $23 \%$ increase (that cannot be explained by just inflation).

In 2015, the lowest private sector price was in India, via laboratories in a network called the Initiative for Promoting Affordable and Quality TB Tests (IPAQT) [11]. IPAQT, a private sector initiative coordinated by the Clinton Health Access Initiative (CHAI), that has been able to access concessional prices from several manufacturers of WHO-approved diagnostics by agreeing to charge patients no more than agreed upon ceiling prices. Laboratories in IPAQT offered Xpert at a fixed price of INR 2000 (US\$30.26 in 2015), compared with an average of $\$ 52.82$ in the rest of the private market in India in 2015. The 2017-18 data show a similar trend, with IPAQT laboratories still offering the lowest price (US \$33.80) among all 12 countries. Interestingly, the gap between IPAQT and market prices in India has narrowed between 2015 and 2018, suggesting that IPAQT might have played a role in increasing affordability in the private sector at large.

IPAQT now includes 200 accredited, private laboratories, and since 2013, these laboratories have conducted nearly 700,000 WHOendorsed TB tests (including Xpert MTB/RIF, line probe assays, and liquid cultures) at negotiated prices (Harkesh Dabas, CHAI, India, personal communication). Several test manufacturers are now engaged in IPAQT, underscoring their willingness to partner with non-profits and global health agencies.

In addition to IPAQT, there are other approaches to increasing access to new tools. For example, the pediatric TB partnership between India's Revised National TB Control Programme (RNTCP) and Foundation for Innovative New Diagnostics (FIND), increased high-quality testing for children in the private sector in India, by leveraging public sector GeneXpert facilities for testing pediatric samples referred from the private 
Table 1 Prices paid by private patients for Xpert MTB/RIF in 12 high burden countries in 2015 and 2017-18

\begin{tabular}{|c|c|c|c|c|c|c|}
\hline Country & $\begin{array}{l}\text { Mean price } \\
\text { for Xpert MTB/ } \\
\text { RIF } 2015\end{array}$ & $\begin{array}{c}\text { Mean price } \\
\text { for Xpert MTB/ } \\
\text { RIF 2017-18 }\end{array}$ & Range 2015 & Range 2017-18 & $\begin{array}{l}\text { Labs contacted } \\
\text { in } 2015 \text { with } \\
\text { Xpert testing }\end{array}$ & $\begin{array}{l}\text { Labs contacted } \\
\text { in } 2017-18 \text { with } \\
\text { Xpert testing }\end{array}$ \\
\hline Kenya & $\$ 80.60$ & $\$ 85.36$ & $\$ 51-\$ 171$ & $\$ 58.20-\$ 149.38$ & 5 & 5 \\
\hline \multicolumn{7}{|l|}{ India } \\
\hline IPAQT$^{\star}$ member laboratories & $\$ 30.26$ & $\$ 33.80$ & Fixed Price & Fixed Price & - & - \\
\hline Rest of Private Sector & $\$ 52.82$ & $\$ 46.70$ & $\$ 27.84-\$ 86.55$ & $\$ 24.67-\$ 80.19$ & 13 & 22 \\
\hline Pakistan & $\$ 37.26$ & $\$ 47.67$ & $\$ 25.96-\$ 58.65$ & $\$ 25.63-\$ 66.45$ & 4 & 7 \\
\hline Philippines & $\$ 155.44$ & $\$ 152.49$ & $\$ 128-\$ 183$ & $\$ 106.4-\$ 170$ & 9 & 8 \\
\hline Bangladesh & $\$ 74.75$ & $\$ 64.20$ & $\$ 45.50-\$ 130$ & $\$ 42-\$ 90$ & 4 & 6 \\
\hline Afghanistan & $\$ 50.00$ & No Xpert & - & - & 1 & - \\
\hline Uganda & No Xpert & No Xpert & - & - & - & - \\
\hline Vietnam & No Xpert & No Xpert & - & - & - & - \\
\hline Indonesia & No Xpert & No Xpert & - & - & - & - \\
\hline Myanmar & No Xpert & $\$ 71.03$ & - & - & - & 1 \\
\hline Nigeria & No Xpert & $\$ 175.00$ & - & $\$ 115.00-\$ 235.00$ & - & 2 \\
\hline Cambodia & No Xpert & No Xpert & - & - & - & - \\
\hline
\end{tabular}

Greater than $50 \%$ of all primary health-care visits in the countries listed in this table were to a private healthcare provider [6]. Prices correct at December 2017. ${ }^{\star}$ IPAQT refers to the Initiative for Promoting Affordable and Quality TB Tests (www.ipaqt.org), a private sector initiative coordinated by the Clinton Health Access Initiative in New Delhi, which offers WHO-approved diagnostics at concessional prices.

sector [12]. This strategy needs to be scaled-up for both adult and pediatric TB testing, to ensure public GeneXpert facilities, which have excess capacity, are optimally used for greatest impact [13].

Overall, our data show that while Xpert is now available in more countries in the private health sector, it largely remains expensive for patients. It is important to acknowledge that high price is not entirely driven by test manufacturers. In addition to higher commercial prices by the manufacturer, there are other costs such as shipping and import costs, distributor margins, incentives to doctors, and mark-ups by laboratories and hospitals. Cumulatively, these factors result in the high prices reflected in the surveys. Thus, interventions such as import duty waivers for all essential diagnostics, and tighter regulation (e.g. price controls) by governments to prevent price gouging by private hospitals and laboratories are worth considering.

Our data also illustrate the need for novel private sector business models like IPAQT to increase as well as maintain affordability of new tools. The IPAQT model is now being considered in other highly privatized countries, as manufacturers have indicated willingness to collaborate with local private laboratory networks and partners to expand access. Also, IPAQT recently expanded its menu to include quality-assured tests for HIV and hepatitis C [14], underscoring the importance of leveraging such initiatives to go beyond $\mathrm{TB}$, increase affordability and access to a variety of global health products, and optimize the use of multi-disease platform technologies such as GeneXpert [15,16].

In the longer run, we need more affordable multi-disease platform technologies that are designed for low resource settings, that can be used to deliver a variety of tests included in the Essential Diagnostics List that will be released by World Health Organization this year [17]. Novel technologies coming out of countries such as India and China offer a lot of hope, and could potentially reach a larger population than expensive technologies designed for high income markets.

\section{ACKNOWLEDGEMENTS}

We are grateful to colleagues in 12 countries for sending us information. We thank Harkesh Dabas, Arnab Pal, Manisha Sabharwal, and Rigveda Kadam (CHAI, New Delhi, India), and Lekha Puri (CHAI, Viet Nam) for their helpful input on the analysis. NP and WVG have no competing interests. CMD is employed by FIND, Geneva, which partners with several companies to produce and evaluate global health diagnostics; MP declares no competing interests, but is a consultant to the Bill \& Melinda Gates Foundation, on the Scientific Advisory Committee of FIND, and part of the Governing Council of IPAQT in India, coordinated by CHAI, New Delhi, India.

\section{APPENDIX A. SUPPLEMENTARY DATA}

Supplementary data associated with this article can be found, in the online version, at https://doi.org/10.2991/j.jegh.2018.04.005.

\section{REFERENCES}

[1] World Health Organization. Global Tuberculosis Report 2017. Geneva: WHO; 2017

[2] Hanson C, Osberg M, Brown J, Durham G, Chin DP. Finding the missing patients with tuberculosis: lessons learned from patient-pathway analyses in 5 countries. J Infect Dis 2017; 216;S686-S95.

[3] Wells WA, Uplekar M, Pai M. Achieving systemic and scalable private sector engagement in tuberculosis care and prevention in asia. PLoS Med 2015;12;e1001842.

[4] Sreeramareddy CT, Kishore PV, Menten J, Van den Ende J. Time delays in diagnosis of pulmonary tuberculosis: a systematic review of literature. BMC Infect Dis 2009;9;91.

[5] Mistry N, Lobo E, Shah S, Rangan S, Dholakia Y. Pulmonary tuberculosis in Patna, India: durations, delays, and health care 
seeking behaviour among patients identified through household surveys. J Epidemiol Glob Health 2017;7;241-8.

[6] Mistry N, Rangan S, Dholakia Y, Lobo E, Shah S, Patil A. Durations and delays in care seeking, diagnosis and treatment initiation in uncomplicated pulmonary tuberculosis patients in Mumbai, India. PLoS ONE 2016;11;e0152287.

[7] Cazabon D, Alsdurf H, Satyanarayana S, et al. Quality of tuberculosis care in high burden countries: the urgent need to address gaps in the care cascade. Int J Infect Dis 2016.

[8] Pai M, Furin J. Tuberculosis innovations mean little if they cannot save lives. Elife 2017;6;e25956.

[9] World Health Organization. Policy update: automated real-time nucleic acid amplification technology for rapid and simultaneous detection of tuberculosis and rifampicin resistance: Xpert MTB/RIF system for the diagnosis of pulmonary and extrapulmonary TB in adults and children 2013. http://www.stoptb.org/ wg/gli/assets/documents/WHO\%20Policy\%20Statement $\% 20$ on\%20Xpert\%20MTB-RIF\%202013\%20pre\%20publication\%20 22102013.pdf (accessed).

[10] Puri L, Oghor C, Denkinger CM, Pai M. Xpert MTB/RIF for tuberculosis testing: access and price in highly privatised health markets. Lancet Global Health 2016;4;e94-e5.

[11] Clinton Health Access Initiative. Case study. Catalyzing the market for accurate tuberculosis testing in India's extensive private sector through IPAQT. https://clintonhealthaccess.org/ content/uploads/2016/06/Case-Study-India-IPAQT-June-2016. pdf 2016. (accessed 10.04.18.).

[12] Raizada N, Khaparde SD, Salhotra VS, et al. Accelerating access to quality $\mathrm{TB}$ care for pediatric TB cases through better diagnostic strategy in four major cities of India. PLoS ONE 2018;13;e0193194.

[13] Salje H, Andrews JR, Deo S, et al. The importance of implementation strategy in scaling up Xpert MTB/RIF for diagnosis of tuberculosis in the indian health-care system: a transmission model. PLoS Med 2014;11;e1001674.

[14] Mascarenhas A. Diagnostic lab group in India to offer HCV and HIV tests at almost half the price. 2018. http://indianexpress. com/article/india/diagnostic-lab-group-in-india-to-offer-hcvand-hiv-tests-at-almost-half-the-price-5090239/. Indian Express.

[15] Ndlovu Z, Fajardo E, Mbofana E, et al. Multidisease testing for HIV and TB using the GeneXpert platform: a feasibility study in rural Zimbabwe. PLoS ONE 2018;13;e0193577.

[16] Nash M, Ramapuram J, Kaiya R, Huddart S, Pai M, Baliga S. Use of the GeneXpert tuberculosis system for HIV viral load testing in India. Lancet Global Health 2017;5;e754-e5.

[17] World Health Organization. WHO to develop Essential Diagnostics List. 2017. http://www.who.int/medicines/news/2017/WHO_ develop_essential_diagnostics_list/en/; (accessed 21.04.18.).

\title{
Nirros Ponnudurai
}

Faculty of Medicine, McGill University, Montreal, Canada

\section{Claudia M. Denkinger}

Foundation for Innovative New Diagnostics, Geneva, Switzerland

\author{
Wayne Van Gemert \\ Global Drug Facility, Stop TB Partnership, Geneva, Switzerland
}

\section{Madhukar Pai*}

McGill International TB Centre, McGill University, Montreal, Canada

Manipal McGill Centre for Infectious Diseases, Manipal Academy of Higher Education, Manipal, India

* Address: McGill University, Dept of Epidemiology \& Biostatistics,

1020 Pine Ave West, Montreal, QC H3A 1A2, Canada.

E-mail address: madhukar.pai@mcgill.ca

Received 24 April 2018

Accepted 25 April 2018 